\title{
Distortion Minimization: A Framework for the Design of Plane Geometric Anamorphosis
}

\author{
Vesna Stojakovic $^{1} \cdot$ Bojan Tepavcevic $^{1}$
}

Published online: 9 May 2016

(C) Kim Williams Books, Turin 2016

\begin{abstract}
Anamorphosis, as a drawing, represents shapes on a surface such that they appear in their natural form only under specific viewing conditions. Although anamorphoses are mainly studied in a historical context, they are currently experiencing a revival. Plane geometric anamorphoses are a specific sub-type of anamorphic drawings. Some practical problems may arise during the design and realization of plane geometric anamorphosis causing the 3D illusionistic effect to be impaired. The aim of this paper is to identify and analyze these problems. In the paper we use parametric analysis to quantify the distortion that may appear because the point of view is offset from the preferred point of view, and to simulate the deviations that can appear because of the errors in onsite realization. The analysis leads to a framework for the design of plane geometric anamorphosis that minimizes the impairment of the anamorphic illusion.
\end{abstract}

Keywords Anamorphosis · Geometry · Perspective · Parametric analysis

\section{Introduction}

The human visual system relies on retinal $2 \mathrm{D}$ images to perceive a $3 \mathrm{D}$ shape. The relationship between the information in the images perceived by the retinas and their real-world sources may cause shape ambiguity (Pizlo 2008, pp. 21-27; Schröter 2014, pp. 38-51). In the perception of geometry, the spatial properties of $3 \mathrm{D}$ objects are projected onto a plane. Hence, people often do not experience a

Vesna Stojakovic

vesna100@uns.ac.rs

1 Department of Architecture and Urbanism, Faculty of Technical Sciences, University of Novi Sad, Trg Dositeja Obradovica 6, 21000 Novi Sad, Serbia 
veridical representation of the space surrounding them. This phenomenon is often referred to as an 'illusion' (Howe and Purves 2005, pp. 4-24).

Anamorphosis is an illusion that is intentionally created in order to simulate a virtual spatial structure. It represents shapes such that they appear in their natural form only under specific viewing conditions (an unusual position of the observer, direction of viewing, or the reflection from a curved mirror are the most common scenarios). While there is no difference between anamorphosis and a perspective image in the geometric construction, the perception of a plane anamorphic picture differs from the perception of a perspective image. The crucial difference is that the anamorphic picture is distorted and to see the 3D illusion the direction of the observer's view has to be oblique to the plane in which the picture is drawn.

\section{Development of Plane Anamorphic Pictures}

Anamorphic pictures appear in European art and architecture shortly after the development of linear perspective geometric constructions in the period of the Renaissance (Veltman 1986, pp. 2-21). Anamorphoses were used in many different forms and for different reasons, most often to simulate non-existing spaces or to transmit secret messages (Hagi 2002, pp. 68-69; Castillo 2001, pp. 1-17). One of the most famous historical examples of anamorphic pictures, The Ambassadors by Hans Holbein, has been studied by many authors (Sharp 1998, pp. 157-165; Hagi 2002, pp. 61-68; Collins 1992, pp. 73-80).

Considering the techniques used for anamorphic construction, one of the methods used to create anamorphic drawings on a plane is to map a grid of squares onto a distorted grid and transfer each piece of the picture onto its corresponding field. This method was introduced by Jean François Niceron in his book La perspective curieuse ou magie artificielle des effets merveilleux written in 1638, where he presented a set of simple geometric rules on how to create anamorphoses that could be used to guide laymen who are not acquainted with geometry (Andersen 2007, pp. 452-457; Baltrusaitis 1977, pp. 57-60; Pérez-Gómez and Pelletier 1977, pp. 142-143). The anamorphic drawing created using this method is shown in Fig. 1. Use of similar grid construction approaches in perspective drawings and anamorphosis have remained popular and are still studied today, since this method is suitable for many interesting applications (Sharp 2010, pp. 7-10; Reynolds 2003, pp. 138-155; García-Salgado 2003, pp. 22-48; Hansford and Collins 2007, 2012; Hunt et al. 2000, pp. 232-237). On the other hand, the grid construction methods are not always the best solution to transfer the anamorphic picture. Depending on the method used for realization, deviations that can appear as a consequence of imprecision may be more visible when grid construction is used because the relationship between projection elements is not taken into consideration.

Although anamorphoses are mainly studied in a historical context (Pérez-Gómez and Pelletier 1977; Andersen 2007; Massey 2007, pp. 124-126), they are currently experiencing a revival (Topper 2000, pp. 115-124) and again finding favor, probably due to the ease of producing anamorphic images using computer graphics modeling (Di Paola et al. 2015, pp. 253-285; Hansford and Collins 2007, pp. 214-222; Solina and Batagelj 2007, pp. 1-4; De Comite 2011, pp. 33-38, 


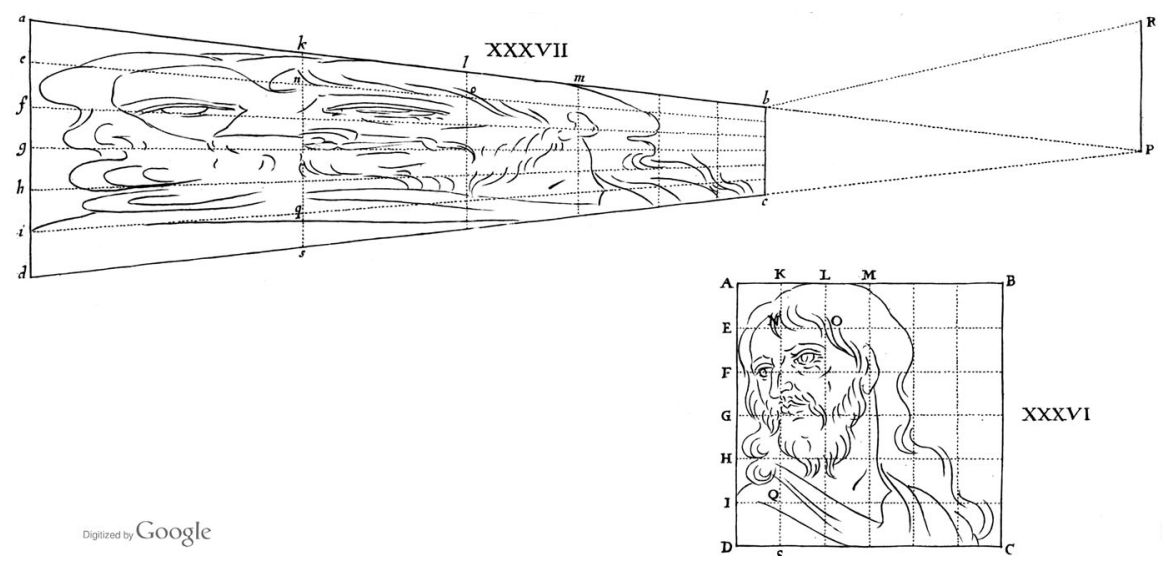

Fig. 1 Anamorphic drawing created by the grid construction method. Niceron, La Perspective Curieuse, p. 241 (Google digital content, http://www.books.google.com)

Čučaković and Paunović 2015, p. 620), and the strong illusionistic effects which may be created. This study is different from other recent and relevant research on anamorphosis because it quantifies distortion and deviation for the specific type of anamorphic pictures using parametric analysis and simulations.

Anamorphic images appear in a variety of media such as architectural and urban design, industrial design, scenic design, and art for the purposes of revitalization, decor, advertising as well as other related areas. Its application varies in scale from smaller examples in fine art to the pictures that occupy large urban spaces. Anamorphoses can be drawn on complex surfaces or spread over several different planes. In the urban space, anamorphoses are most often drawn onto the horizontal plane, since in the urban space wide areas, such as streets and squares, are ideal for creating an illusionistic anamorphic effect for an observer standing at ground level. Such anamorphoses, created in an urban context became extremely attractive with the development of social networks since the urban locations of the anamorphoses allow people to interact with the image and the illusionary space and make creative photographs.

\section{Plane Geometric Anamorphoses}

In this paper we discuss plane geometric anamorphoses. The term geometric anamorphosis is used in this paper to refer to a particular style of picture, an anamorphic picture with geometrical motifs, which consists of solid color areas shaped like geometric figures.

In this paper we will consider the anamorphic pictures in a single plane (plane anamorphosis). The ranges of parameters are set to be representative of anamorphosis at an urban scale. We assume that the anamorphic image is in the same horizontal plane upon which the observer is standing. The observer can experience the anamorphosis via eyesight or a camera. Binocular vision is ignored 
in this paper, and we assume that the anamorphosis is viewed from a single point (one eye or camera). This point is the observer's point of view.

The observer's point of view needs to be at a specific position in relationship to the picture in order to perceive the illusionary spatial structure. This point of view is the center of the perspective projection and we will refer to it as the preferred point of view. The orthogonal projection of the preferred point of view onto the picture plane is the preferred standpoint. The line that contains the observer's point of view and the point on the picture plane onto which the observer is looking represents the direction of the observer's view and this line is the direction of view.

The importance of the direction of view showing the distinction between an anamorphic and a perspective picture is illustrated in Fig. 2. The perspective projections of two cubes, Cube 1 and Cube 2, are shown in two orthogonal views. The cubes are of the same size and have parallel edges. The observer's point of view $(O)$ and picture plane $(\pi)$ are the same. Only the direction of view $(d)$ differs. If the observer's direction of view $\left(d_{1}\right)$ is perpendicular to picture plane $\pi$ then Cube 1 will be in their visual field and Cube 2 will be outside of it. For Cube 2 to become visible, the direction of view $\left(d_{2}\right)$ has to be oblique in relation to the picture plane $\pi$. Thus, the perspective projection of Cube 2 appears distorted and in this case we consider it an anamorphic drawing.

There are two main reasons that cause the observer to experience an inaccurate shape from an anamorphic projection: point of view offset and picture errors. To distinguish between these two causes of impairment in the illusionistic effect, we will use the terms distortion and deviation. If the observer is offset from the preferred point of view the anamorphic picture will appear distorted to them and we will refer to this mismatch as a distortion of the picture. On the other hand, if the picture is not accurately realized onsite, the mismatch will be referred to as deviation of the picture.

Since an anamorphic image has to simulate $3 \mathrm{D}$ space using a 2D image it is very important to emphasize lines that simulate the direction perpendicular to the picture plane. If such lines are not emphasized then the $3 \mathrm{D}$ effect is impaired and the picture

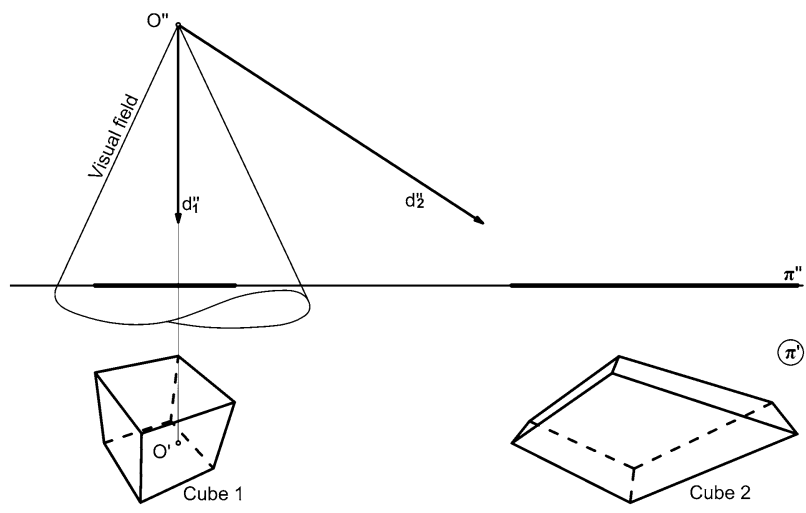

Fig. 2 For Cube 1 the direction of view $\left(d_{1}\right)$ is perpendicular to picture plane $\pi$. For Cube 2 the direction of view $\left(d_{2}\right)$ is skewed 
looks flat. Assuming that the picture plane is horizontal, the main direction to be emphasized in an image is the perspective projection of verticals. Lines that simulate the vertical direction in an anamorphic picture, but actually lie in the picture plane, will be referred to as imaginary verticals. Lines that are perpendicular to the picture plane (lines on the surrounding objects) will be referred to as real world verticals.

\section{Common Problems in Perceiving Geometric Anamorphosis}

The effect of a three-dimensional illusion of anamorphosis depends significantly on the parameters of the picture and on the accuracy of realization. If the design of an anamorphic picture is not planned carefully then the illusion of a $3 \mathrm{D}$ shape can be impaired. The aim of this paper is to identify and analyze problems that need to be considered during the design of a plane anamorphosis in order to successfully create the 3D illusion effect.

If the observer's point of view is offset from the preferred point of view the distortion will be apparent and this is most apparent in the imaginary verticals. Parameters such as the width of the picture, the direction of view and the distance of observer from the picture all influence the intensity of the distortion.

Even if these picture parameters are carefully chosen, errors in realization can cause deviation of geometric anamorphosis. The position of the picture elements and the method of realization both influence apparent deviation in realized pictures.

In this paper a parametric analysis is used in order to provide a quantitative measure of the distortions and deviations of plane anamorphic pictures in relationship to the picture parameters. Such analysis is important because it reveals a methodology that decreases the potential distortion and deviation before realization and thus minimizes the impairment of the $3 \mathrm{D}$ illusionistic effect in the realized picture.

\section{Method}

All necessary analyses were performed in the Rhinoceros environment. We used the Grasshopper plug-in for visual programming in order to perform a quantitative parametric analysis and to simulate the process of realization.

In order to quantify the distortion of the picture that appears because of the point of view offset we created an algorithm that generates automatic measurements of the mismatch between the imaginary verticals and the real world verticals for a given set of parameters. Data that influences the distortion (the offset of the observer's point of view and parameters of the anamorphic picture) was used as input data for the algorithm. The output data was the quantified distortion of the picture as it appears to the observer.

Another algorithm was used to quantify the influence of the errors that may appear during the realization. For each point in the picture plane, the input data is the point offset in the picture plane and the output data is the ratio between the error in the picture plane and the error as seen by the observer. 
Simulating the realization process was done using a third algorithm, which automatically creates random offsets of specified points within the defined range. The result is a deviated anamorphic picture.

\section{Point of View and Geometric Anamorphosis}

Anamorphoses may be used to create an impressive representation of a 3D shape under perfect observing conditions. The dual nature of the visual perception allows us to see a rigid projection and recognize the shape constancy in perspective pictures at the same time (Gibson 1979, pp. 151-160) even if the observer is not in a preferred point of view. In plane anamorphic pictures that are designed to have one preferred point of view the $3 \mathrm{D}$ effect is perceivable only from the point that is close to it.

One of the variations that have to be considered in anamorphoses is that the real point of view (which can be observer's eye or camera) is often not exactly aligned with the preferred point of view. People vary in height and will often slightly move from the predetermined standing location. Offsetting of the viewpoint causes a distorted perception of an anamorphic picture, which impairs the illusion of 3D shape. These distortions can be easily noticeable in geometric anamorphoses because of the domination of regular shapes and straight lines. Therefore, awareness of the intensity of the distortion is very useful during the design stage.

The observer's point of view (eye or camera) can be offset from the preferred point of view in different directions. If the point of view is below the preferred point of view then the image will look shorter in the imaginary vertical direction, and if it is above, it will seem elongated. However, this distortion is usually imperceptible. When the observer's eye moves to the right or to the left from the preferred viewpoint, then the image will seem skewed to one side and this kind of distortion is particularly noticeable. In geometric anamorphosis it is obvious to the observer that all imaginary verticals are skewed to the same side (either to the right or left), and the observer will intuitively move their head/camera towards the preferred point of view. In Fig. 3, distortions that appear when the observer's position is moved up/down or left/right from the preferred point of view are shown. Variations are shown in the same anamorphic picture, which is at a distance of $5 \mathrm{~m}$ from the

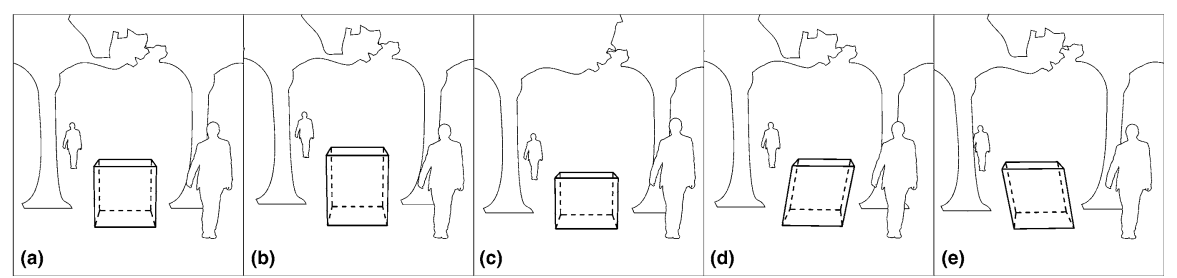

Fig. 3 Anamorphic picture of the cube observed: a from the preferred point of view, $\mathbf{b} 0.3 \mathrm{~m}$ above the preferred point of view, $0.3 \mathrm{~m}$ below the preferred point of view, $\mathbf{d} 0.3 \mathrm{~m}$ to the right the preferred point of view, e $0.3 \mathrm{~m}$ to the left the preferred point of view 
preferred standpoint and is intended to represent a cube ( $1 \mathrm{~m}$ size). The same surroundings are added to each scene, to make the scale and the relationship between imaginary verticals and real world verticals perceivable.

The most critical distortion in viewing occurs when the observer is either in front of or behind the preferred point of view. The problem occurs because the imaginary verticals appear skewed to the observer, which causes the effect of a 3D shape illusion to be impaired. If the direction of view would be parallel to picture plane, then the vanishing point of real world verticals $\left(V_{v 1}\right)$ would be at infinity and verticals would appear as mutually parallel. Since the direction of view $(d)$ is always pointing below the horizon plane, the vanishing point of real world verticals $\left(V_{v 1}\right)$ is below the observer, which is why all verticals appear to intersect below the scene.

If the observer moves forward from the preferred point of view, then the imaginary verticals will appear as if their upper parts are skewed towards the top of the picture. Since all verticals are skewed to the other side (they intersect below the scene) this distortion is not very apparent. A much less favorable scenario occurs when the observer moves back from the preferred point of view. Then the upper parts of the imaginary verticals are skewed towards the bottom of the picture and hence emphasize the skewing effect that appears because the vanishing point of the verticals is below the horizon. This distortion is the most noticeable one, and this is why it will be analyzed in more detail. It is important to plan the anamorphosis carefully during the design stage in order to minimize its effect.

Figure 4 demonstrates what happens when an observer $\left(O_{1}\right)$ stands behind the preferred point of view $(O)$. The picture plane is horizontal and the observer is standing on the picture plane. The anamorphosis is constructed for the preferred point of view $(O)$, and the vanishing point $\left(V_{v}\right)$ of the imaginary verticals $\left(v_{i}\right)$ is at a standpoint of the observer. The vanishing point $V_{v 1}$ of the real world verticals $\left(v_{r}\right)$ is below the vanishing point $V_{v}$, and the mismatch between real world verticals and imaginary verticals becomes apparent.

In order to quantify the distortion the angle between the projection of the imaginary verticals and the projection of real world verticals is measured. The perspective projection is made onto the plane $\gamma(\gamma$ is perpendicular to the direction of view $d$ ) with a center of projection $O_{1}$. The reason why the angle between the imaginary verticals and the real world is measured in perspective projection onto plane $\gamma$ is because we aim to measure the distortion of verticals as the observer sees it. Figure 5 illustrates this concept.

The three main parameters that influence the intensity of the described distortion of the anamorphic picture perception are:

- direction of view,

- width of the picture,

- distance of the picture (or part of the picture).

An algorithm that automatically measures the distortion for the specified point of view offset is shown in Fig. 6. Input data define the picture and distortion parameters. The picture parameters are to be set at the beginning of design. These are the preferred standpoint $\left(V_{v}\right)$, offset standpoint and the height of the eye (distance from the point of view to the picture plane). Distortion input parameters 

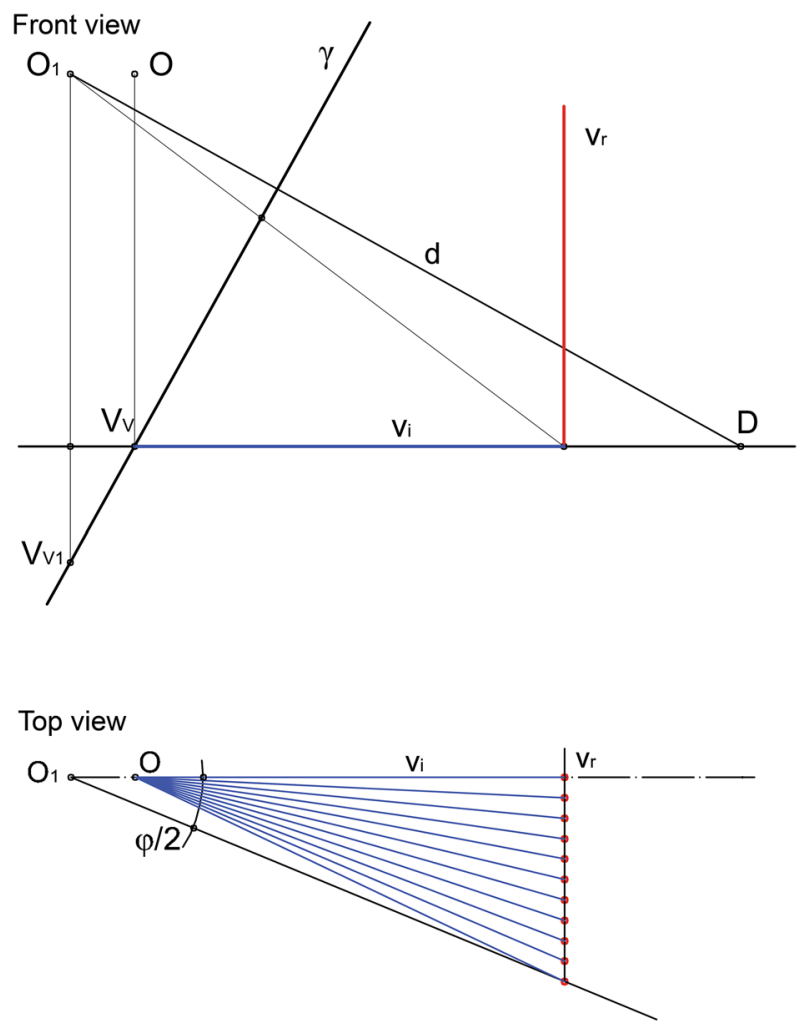

Fig. 4 Point of view offset

are varied in order to analyze the distortion of the picture depending on each of these parameters. The direction of view is defined by the position of point $D$ in the picture plane. The width of the picture is defined by the value of angle $\varphi$. The distance of the picture is defined as the distance of verticals to be analyzed from the preferred standpoint.

The components are organized in clusters in order to achieve a clearer presentation of the systematic approach to algorithm data flow. The point of view $O_{1}$ is constructed, and the offset of this point is limited to between 0 and $0.5 \mathrm{~m}$, because larger non-intentional offsets are not expected. The direction of view $(d)$ is defined as a line that contains the point of view and the point at the picture plane at which the observer is looking $(D)$. We limited the positions of point $D$ to a distance of $2 \mathrm{~m}-100 \mathrm{~m}$ from the preferred standpoint, since we expect the anamorphic picture to be in that area. The width of the picture is defined as the angle of view $(\varphi)$, which can envelop the whole picture. The value of $\varphi$ was limited to values between $0^{\circ}$ and $90^{\circ}$. We included angles up to $90^{\circ}$ because of the wide-angle cameras which are often used for photographing the anamorphic pictures $\left(90^{\circ}\right.$ angle of view corresponds to $21.6 \mathrm{~mm}$ focal length). The distance of the picture is the distance between the real world vertical compared to the imaginary vertical. To set 


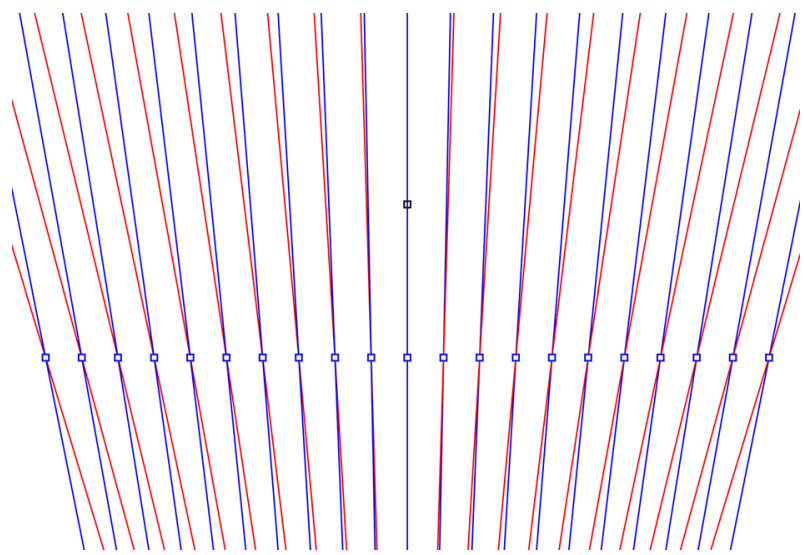

Fig. 5 Distortion from the observer's point of view. Real world verticals are in red, imaginary verticals are in blue (color figure online)

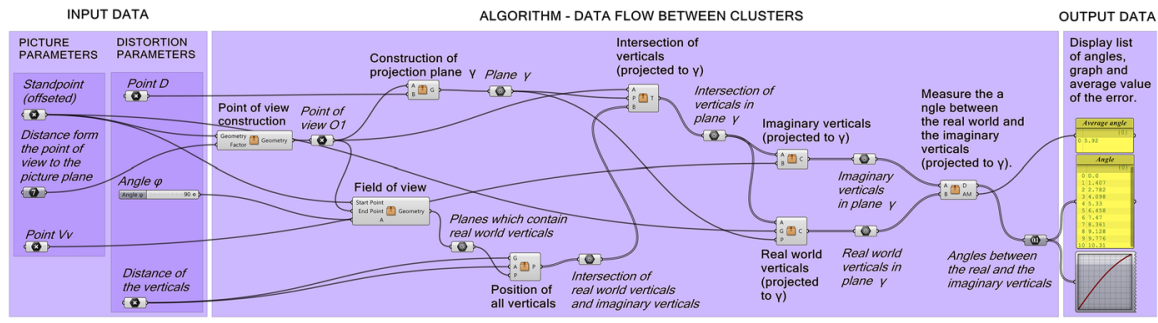

Fig. 6 Concept of Grasshopper algorithm

the distance we use one point in the picture axes (intersection of picture plane and real world vertical $v_{r}$ ). The algorithm calculates the distortion for all verticals that intersect the same line perpendicular to the orthogonal projection of the direction of view to the picture plane. The distance was also limited to between 2 and $100 \mathrm{~m}$, which is expected to contain the entire area of the picture. All parameters and their attributes and ranges described in this paragraph apply to all of the following experiments. For ease of use and applicability of the algorithm in a 3D environment, the input and output data are published to the Remote Control Panel (Fig. 7).

The algorithm uses these input data (position of $O_{1}, \varphi$, position of $D$ and position of vertical $v_{r}$ ) and calculates the list of angles between the perspective projection of real verticals and imaginary verticals to plane $\gamma$. These angles are used to quantify the distortion. For any particular setting of parameter values we can obtain the average angle of distortion in the verticals. We used the average angle instead of a list of angles, because we want to observe distortion for the picture as a whole, as the observer perceives it. By varying one parameter we obtain a range of average distortion values. For any particular parameter the changes made in the average distortion by varying the parameter will be considered an additional measure of 


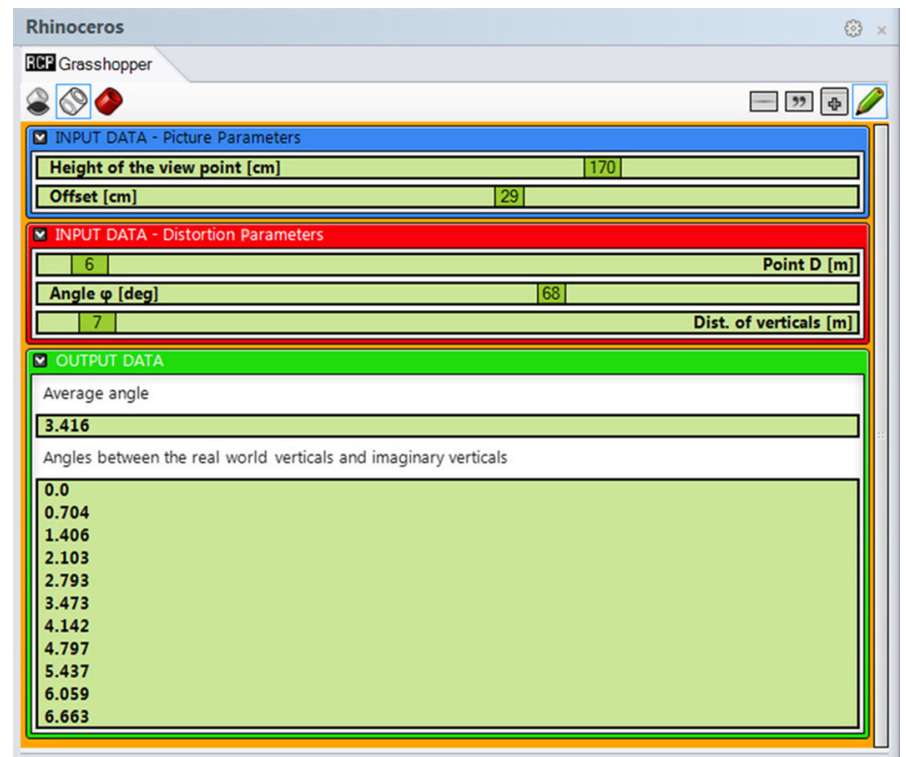

Fig. 7 Input and output data published to RCP

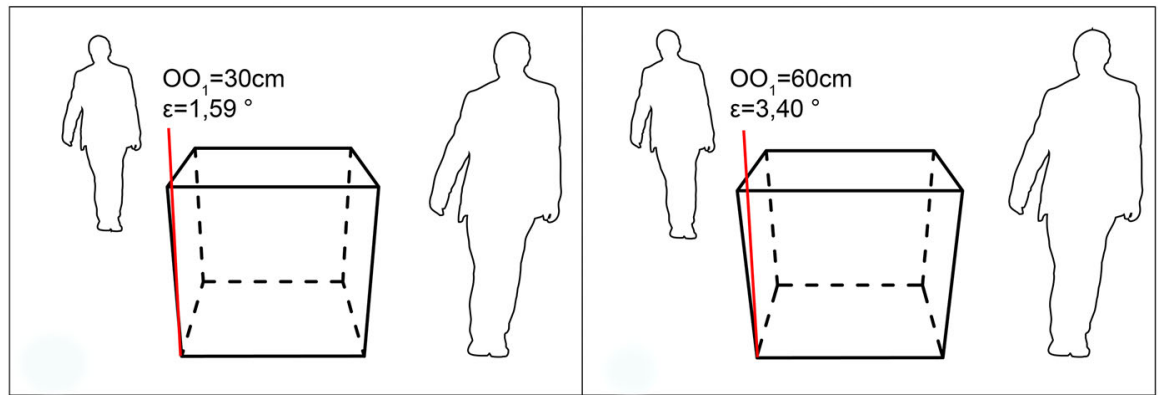

Fig. 8 Distortion $(\varepsilon)$ for different point view offset values (OO1). Anamorphic picture is in black and real world verticals are in red (color figure online)

distortion, with the difference between the smallest and the largest of these the range of distortion.

In order to analyze the influence of each parameter on the distortion, we varied each parameter while the other parameters were set to their default value (default values are: $O_{1}$ is $0.3 \mathrm{~m}$ behind $O, D$ is at a distance of $3.5 \mathrm{~m}, \varphi=50^{\circ}, v_{r}$ is $3 \mathrm{~m}$ from preferred standpoint).

The distortion of verticals that appear skewed becomes progressively more noticeable the further the observer is from the preferred point of view. The same anamorphic picture rendered from a different point of view $\left(O_{1}\right)$, which is behind the preferred point of view $(O)$, is shown in Fig. 8 . 


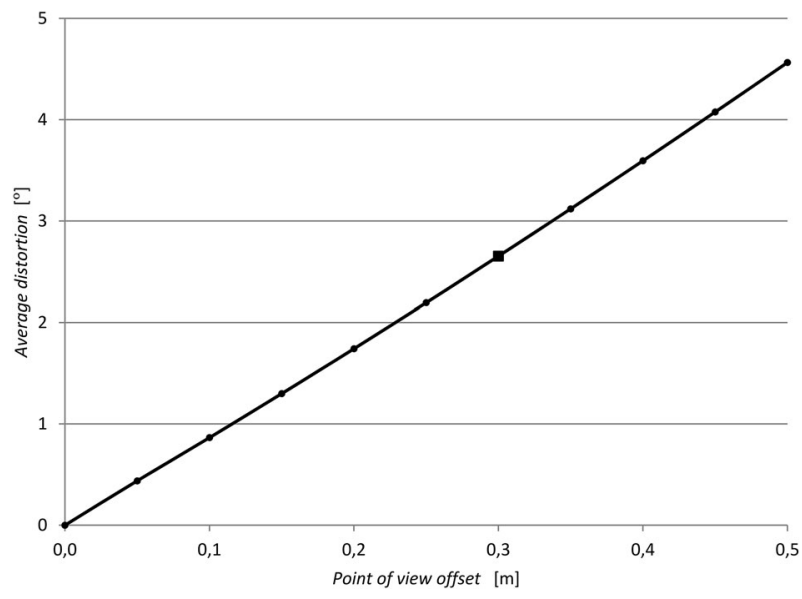

Fig. 9 Average distortion depending on the point of view offset. Default value is marked as square

The point of view offset is not a parameter of the picture, and therefore cannot be planned for, but it is very important to show its influence on the distortion. In Fig. 9 the variation in the point of view offset and its influence to average distortion is shown. All other parameters are set to the default values. The average distortion changes by $4.57^{\circ}$. If all parameters are set to the worst-case scenario (to the values that maximize the distortion while are within the defined limits), the average distortion is $10.57^{\circ}$ and the biggest distortion (of the most left/right vertical) is $19.65^{\circ}$.

Others parameters are the parameters of the anamorphic picture, and therefore can be considered during the design. First, we analyze the variations in the direction of view $(d)$. When the point $D$ is closer to the observer the distortion is larger, because the projection plane $\gamma$ will be more oblique to the picture plane, which increases the distortion. On the other hand, as the point $D$ moves closer to the observer the distance between vanishing points $V_{v}$ and $V_{v 1}$ is larger, which decreases the distortion. Therefore, the relationship between direction of view and the distortion is not simple. The point $D$ is varied, and the value of the average distortion is shown in Fig. 10. Note that maximum distortion appears around a distance of $4.1 \mathrm{~m}$. More important is to notice that the range of distortion is small $\left(0.22^{\circ}\right)$. Even in the worst-case scenario the range of average distortion is $1.01^{\circ}$ and the range of biggest distortion is $3.91^{\circ}$, which indicates low influence of the direction of view to the distortion in the anamorphic picture when all other parameters are constant.

Next, we will analyze the variations of the angle of view $\varphi$. Middle verticals (the ones on the picture axis) always appear vertical, but imaginary verticals near the side of the picture can appear skewed which can be seen in Fig. 11, in which the distortion for different values of $\varphi$ is presented. The angle of view $\varphi$ is varied and the influence on the average distortion is shown in Fig. 12. We can notice that the width of the picture strongly influences the distortion. If all default values are used 


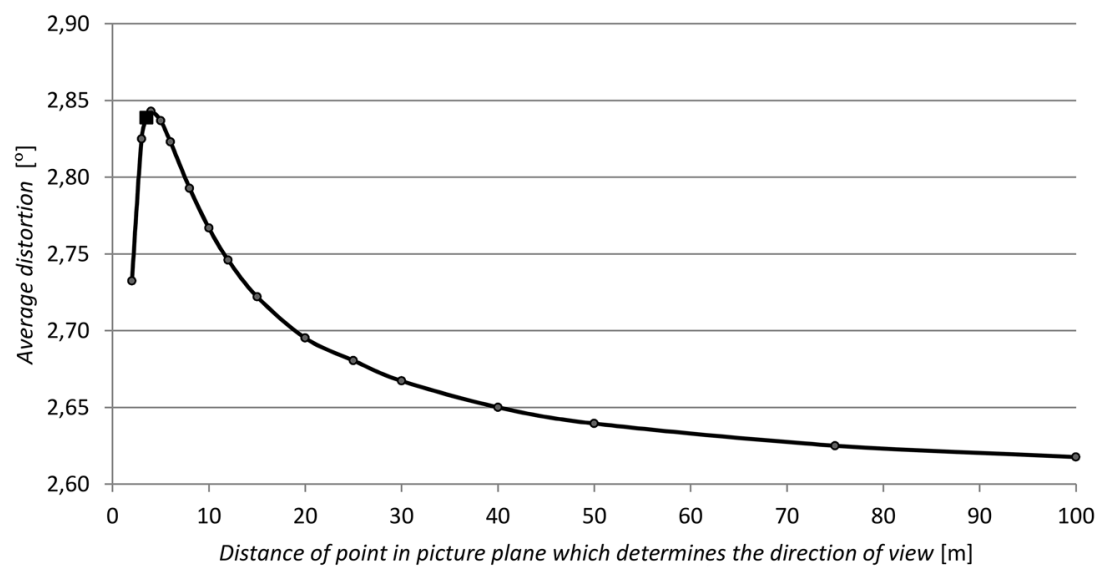

Fig. 10 Average distortion in dependence of $D$. Default value is marked as square

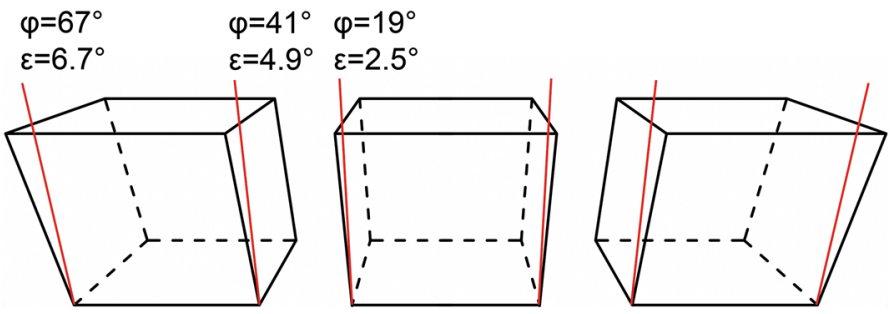

Fig. 11 Distortion $(\varepsilon)$ for different values of $(\varphi)$. Anamorphic picture is in black and real world verticals are in red. Observer is $0.5 \mathrm{~m}$ behind the preferred standpoint (color figure online)

then the average distortion is $5.37^{\circ}$. In the worst case scenario the average distortion is $9.55^{\circ}$, and the biggest distortion is $15.51^{\circ}$. If we limit the angle to the human visual field $\left(\varphi \leq 50^{\circ}\right)$, the average distortion is $2.78^{\circ}$, and in worst case scenario the average distortion is $5.45^{\circ}$, and the biggest distortion is $10.29^{\circ}$.

The third parameter that influences the distortion is the position of the real world vertical. If the vertical $v_{r}$ is closer to the observer, the distortion will be larger and more apparent. We varied the orthogonal distance of the vertical from the preferred standpoint and compared it to the average distortion. The results are shown in Fig. 13. The range of average distortion is $1.17^{\circ}$. In the worst-case scenario the range of average distortion of $1.55^{\circ}$, and the range of biggest distortion is $2.79^{\circ}$.

This parametric analysis is important because the distortion of the skewing verticals can be avoided during the design stage of the project if the designer takes into account the relationship between the picture parameters, such as width of the picture, distance of the picture to the observer and picture's point of interest which guide the direction of view. The algorithm can be used for any plane anamorphic picture to check the distortion for an expected point of view offset. Non-optimal parameters can be compensated by another parameter, in correspondence to the relative distortion range. 
Fig. 12 Average distortion in dependence of $\varphi$. Default value is marked as square

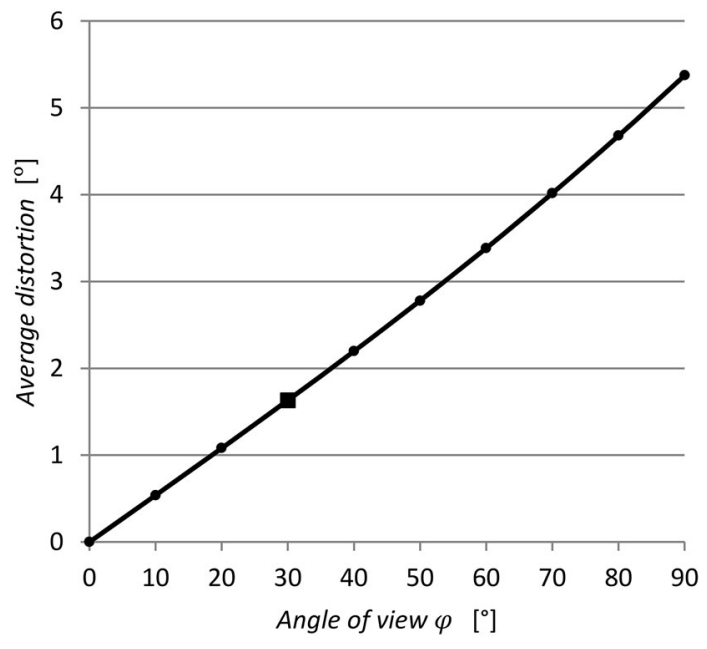

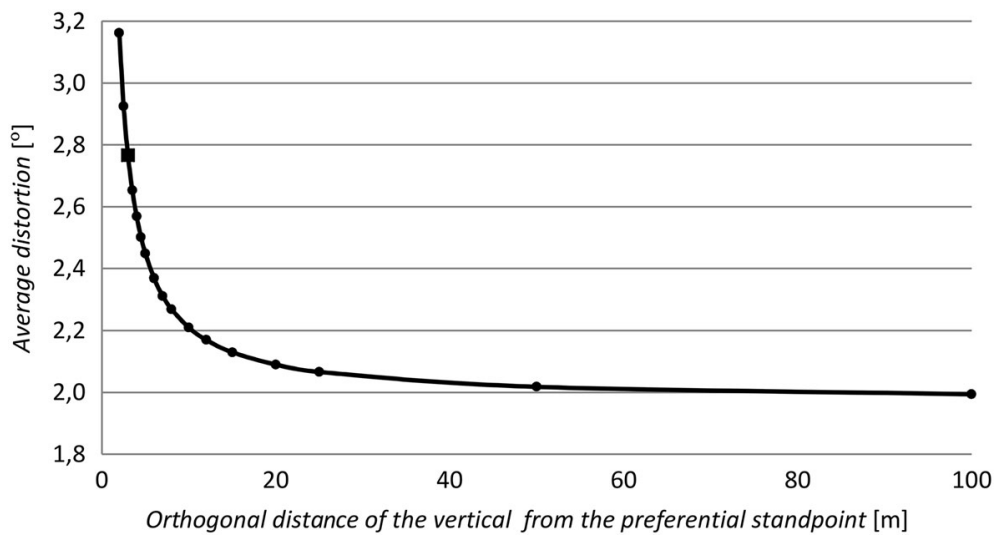

Fig. 13 Average error in dependence of $\left(v_{r}\right)$ distance. Default value is marked as square

\section{Project Realization}

When the design stage is complete, even if the project is designed to avoid distortions, inaccuracy of the anamorphic picture may appear. Onsite errors of a few centimeters in the picture plane are very common for large scale anamorphoses. Deviations that emerge onsite during the project realization in this way can occur due to the method used and/or due to the precision of the realization.

Transformation of the concept from a small scale to a large scale (especially spaces such as an urban space) using the grid construction method may be inaccurate and may not provide satisfactory results for geometric anamorphosis, because geometrical relationships can easily be disrupted. Accurate construction onsite with the use of the vanishing points and other characteristic picture elements 

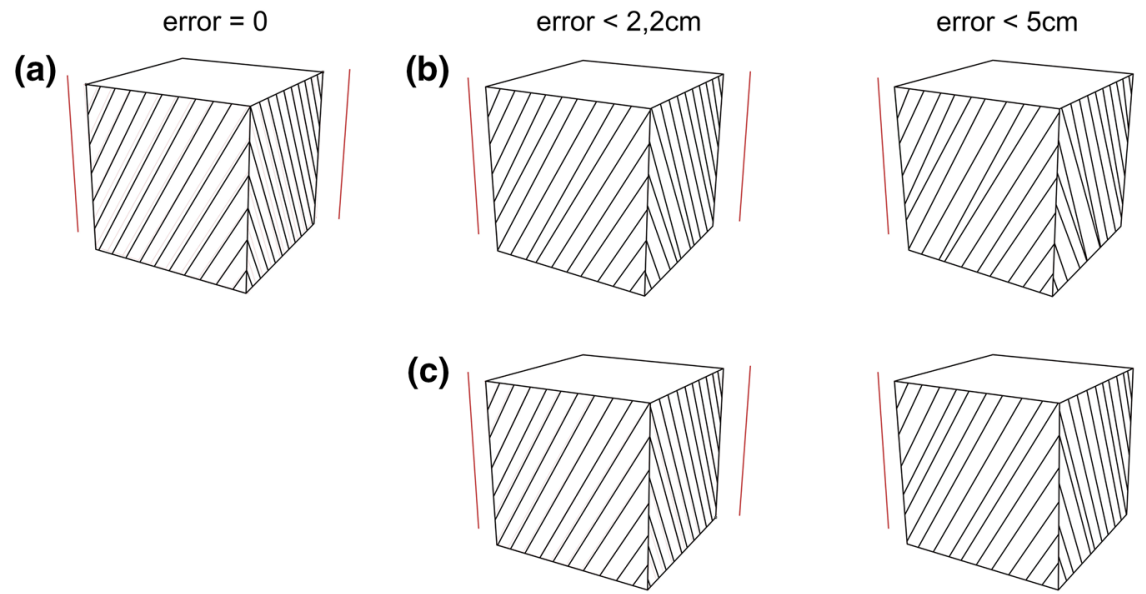

Fig. 14 Simulation of different approaches of realization of anamorphic picture: a project realized without error (b) project realized by grid construction method (c) project realized by vanishing point construction method. Red lines are real world verticals (color figure online)

provides results in which the errors in geometric relationships, such as parallelism, are less apparent.

In Fig. 14 an example of a simulation of two different methods for project realization of an anamorphic picture of a hatched cube are shown. The methods shown are the grid construction method and the construction of vanishing points method. All drawings simulate a cube of $1 \mathrm{~m}$ size, and the drawing in the picture plane is $7.5 \mathrm{~m} \times 3.4 \mathrm{~m}$. In both approaches we assume that the realization precision is the same, which means that the error in the picture plane during the construction of a point is a random value within the defined range. To simulate and compare the results of different approaches the algorithm that moves each point randomly in the picture plane within the given range is used. If the grid construction method is used (Fig. 14b), lines are constructed by connecting the corresponding vertices. When the offset error range is not zero, lines will not intersect in their respective vanishing points, and therefore will not appear parallel. If the vanishing points are constructed first (Fig. 14c), the error of the point construction will be much less apparent, although each point has same error range as in the previous case. We can notice that if the error of each point during construction is $<2.2 \mathrm{~cm}$, the grid construction method hatch lines do not seem parallel in some places, and using the vanishing point construction method the deviation is hardly noticeable. With the error $<5 \mathrm{~cm}$, the deviation is apparent in both cases, but using the grid construction method highly impairs the parallelism of the hatch. Using the vanishing point construction method the hatch is not regular but lines still appear parallel.

Another type of picture deviation is caused by imprecision during the realization. Such errors cause the offset of the points in the picture plane and the bending of the straight lines. In this case the distance of the point offset in the picture plane appears smaller to the observer. The factor of shortening $(r)$ represents the ratio between the length of the line in the real world and the length as it appears to the observer. The 
factor of shortening depends on the direction of view, position of the point, and offset direction. The factor of shortening is maximal if the angle between the direction of view and the picture plane is the biggest, the point is close to the point of view, it is away from picture axes, and if the offset direction is perpendicular to the line that connects the point with the standpoint. The area of low offset shortening is not favorable for placing small details in an anamorphic picture, because the point offset error can be noticeable.

The factor of shortening is illustrated in Fig. 15. The grid of points $(0.2 \mathrm{~m} \times 0.2 \mathrm{~m})$ in the picture plane is shown in the top view. For each point twelve offset directions are marked. The offset shortening factor of each line is presented by the different shade of gray.

Another consequence of the imprecision during the realization is line bending. During realization of a large anamorphosis, it is sometimes hard to construct a perfectly straight line onsite. The appearance of a bulge acts similarly to the point offset as described in the previous section. Besides the shortening factor of the bulge, the shortening factor of the line (the ratio between the length of the line in the picture plane and the line length as it is seen by an observer) influences the appearance of the bending deviation. This factor acts in a similar way to the

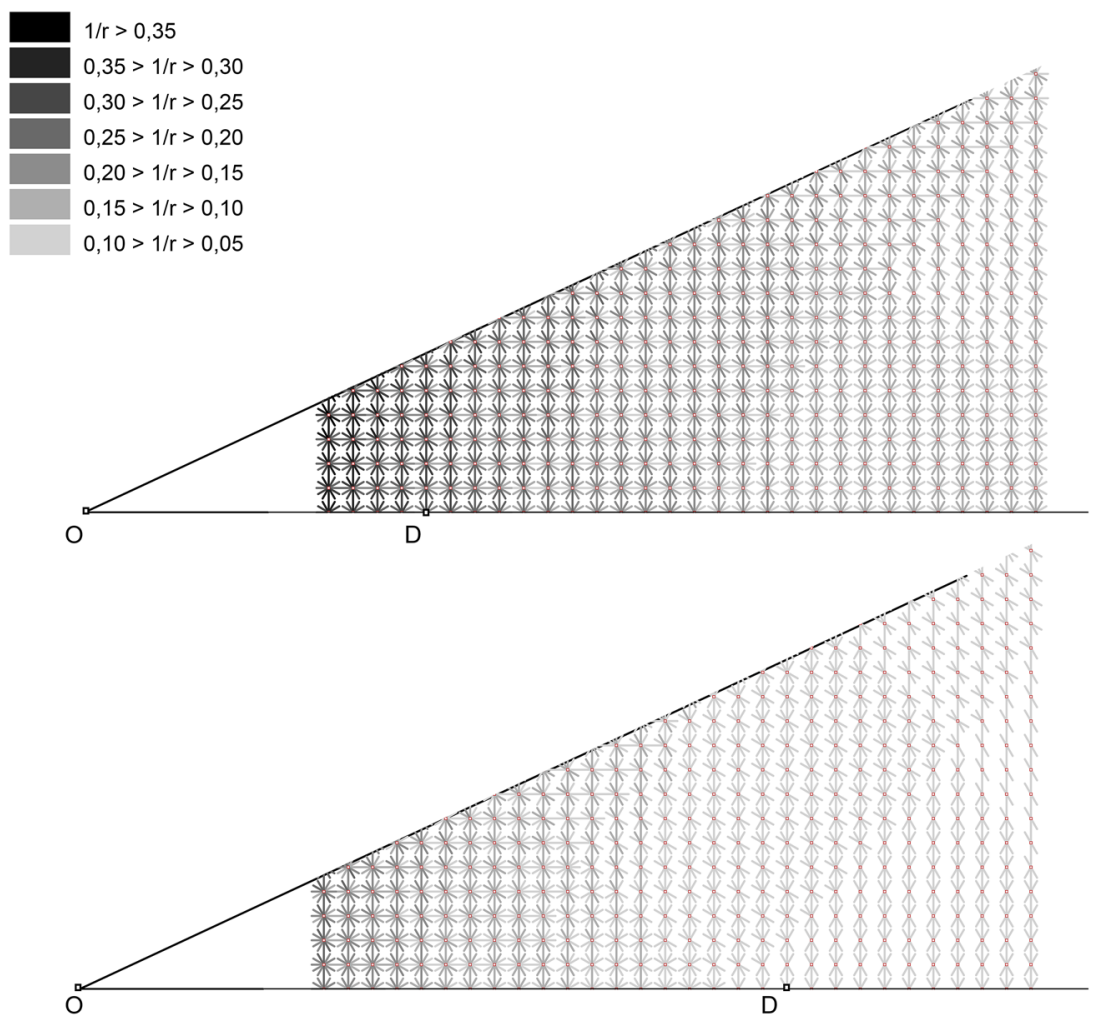

Fig. 15 Intensity of factor of shortening rendered in a top view 
previous case, which is why it will not be taken into further consideration. Lines have a potential for bending if the shortening factor of the line is large, and the shortening factor of the bulge is small. Therefore if the anamorphosis is to be realized using a low precision method then long and distant imaginary verticals should be avoided.

\section{Case Studies}

The detection of major problems described in the previous sections is based on the experiences in realization of urban space geometric anamorphoses during the period between 2010 and 2015. In this section, we present three case studies and use them to illustrate some of the problems and issues previously discussed in this paper as they were encountered in practice.

\section{Amphitheater}

In the design of this project the constraints that influence the skewing of verticals were not considered. The angle of view is $\varphi=60^{\circ}$, distance of the closest point of the picture is $3 \mathrm{~m}$ and distance of $D$ is around $4 \mathrm{~m}$. These parameters caused a high risk for distortion to appear in the case of point of view offset. The noticeable skewing of the imaginary verticals, especially in the left/right side of the image can be seen in Fig. 16a.

In the realization the vanishing points method was used. Arches were constructed onsite using the coordinates of the center and radius. The realization of the project did not cause any deviations.

\section{Cube}

In the design of this project possible skewing of the verticals was taken into consideration and the parameters $\left(\varphi=17^{\circ}\right.$, distance of the closest point of the picture is $3 \mathrm{~m}$ and distance of $D$ is around $4.5 \mathrm{~m}$ ) were chosen to minimize the potential distortion.

In realization the vanishing points methods was used. This was significant for this project because at the end of the realization huge point offsets, up to $20 \mathrm{~cm}$, appeared. In spite of this, the deviation is not obvious in the realized project (Fig. 16b).

\section{City Vistas}

In the design of this project the possible skewing of verticals was considered and the parameters $\left(\varphi=19^{\circ}\right.$, distance of the closest point of the picture is $5 \mathrm{~m}$ and distance of $D$ is around $10 \mathrm{~m}$ ) were chosen to minimize the potential for distortion. Using these parameters the distortions were not obvious even with the large point of view offsets. 
(a)

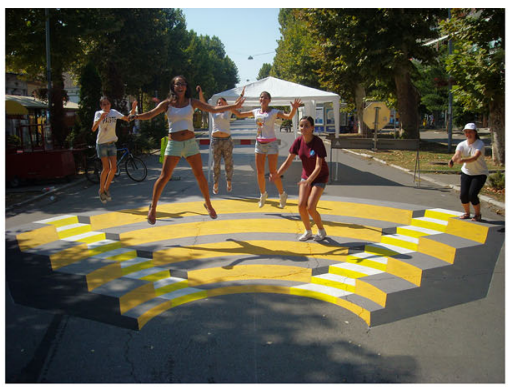

(b)

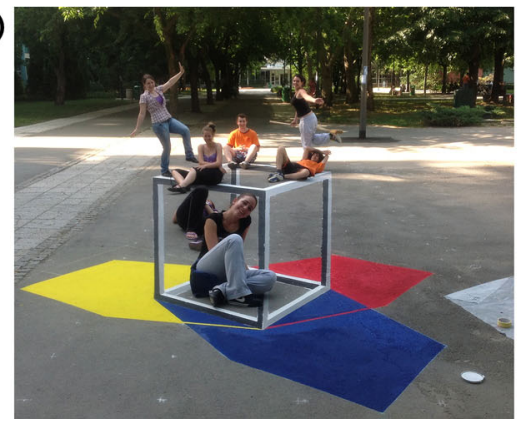

(c)

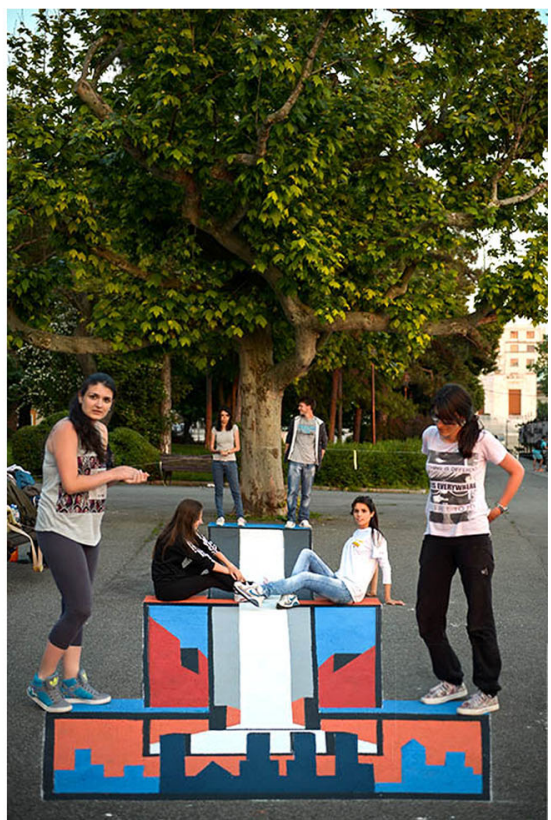

Fig. 16 Examples of realized geometric anamorphosis in urban space: a amphitheater, $\mathbf{b}$ cube, $\mathbf{c}$ city vistas

During realization the vanishing points method was used, so the imaginary parallelism was not impacted. However, high deviations appeared during realization. The reasons for the deviations were small details in the area with a small shortening factor and imaginary verticals with high shortening factor. The point offset and bending are noticeable in the final project (Fig. 16c).

\section{Conclusion}

In the design and realization of plane geometric anamorphosis it is important to consider parameters that may cause distortions and/or deviations of the picture because this can lead to the impairment of the $3 \mathrm{D}$ effect. In this paper we have identified and analyzed several problems that cause distortion stemming from an offset point of view and the deviations that can appear during the realization of the project.

The distortion of an anamorphic picture appears when the point of view is offset from the preferred point of view. The situation in which the point of view is behind the preferred point of view is studied in detail because this offset impairs the 3D illusion the most. The parametric analysis revealed that the parameter with the strongest influence on the appearance of this kind of distortion is the width of the picture. The position of the vertical has less influence and the direction of view has the least influence on this kind of distortion. To avoid a mismatch between the 
imaginary verticals and the real world verticals picture parameters should be considered during the design stage of an anamorphic picture.

Deviations in an anamorphic picture appear because of imprecision during the realization. By simulating different methods of realizing geometric anamorphoses with emphasized imaginary parallelism with the same range of error in realization we have shown that the construction of vanishing points onsite is recommended in order to avoid impairment of parallelism.

The analysis of the parameters that influence the shortening factor can be used in preventing deviation caused by point offset and line bending. This analysis points out the areas of the picture that are least favorable for small details and the characteristics of the lines that are in a high risk of bending deviation.

The limitations of this study are that problems discussed in this paper are general issues and concerns. Since each design is different it is impossible to cover all the possible potential problems and provide adequate solutions. The parametric analysis and simulations described in this paper may need to be revised in order to apply them to other individual projects according to their specific concerns and constraints. This paper analyses the anamorphic picture as a perspective projection, and hence ignores binocular vision. Binocular vision can impair the 3D illusion, especially at small distances. We also only consider anamorphoses drawn on the horizontal plane, and similar studies can be applied to anamorphoses drawn on other projection planes and/or multiple planes, which are considered future directions of this work.

Acknowledgments We would like to thank the reviewers for valuable comments and suggestions that improved our paper. This research was supported by the Ministry of Education, Science and Technological Development of the Republic of Serbia (OI174012).

\section{References}

Andersen, K. 2007. The Geometry of an Art-The History of the Mathematical Theory of Perspective from Alberti to Monge. New York: Springer.

Baltrusaitis, J. 1977. Anamorphic Art. New York: Harry N. Abrams, Inc.

Castillo, D. 2001. Awry Views: Anamorphosis, Cervantes, and the Early Picaresque. Lafayette: Purdue University Press.

Collins, D. 1992. Anamorphosis and the Eccentric Observer. Leonardo Journal 25(1): 73-80.

Čučaković, A., and M. Paunović. 2015. Cylindrical Mirror Anamorphosis and Urban-Architectural Ambience. Nexus Network Journal 17: 605-622.

De Comite, F. 2011. A New Kind of Three-Dimensional Anamorphosis. In: Bridges 2011: Mathematics, Music, Art, Architecture, Culture, 33-38.

Di Paola, F., P. Pedone, L. Inzerillo, and C. Santagati. 2015. Anamorphic Projection: Analogical/Digital Algorithms. Nexus Network Journal 17: 253-285.

Gibson, J. 1979. The Ecological Approach to Visual Perception. New York: Lawrence Erlbaum Associates.

García-Salgado, T. 2003. Distance to the Perspective Plane. Nexus Network Journal 5(1): 22-48.

Hagi, K. 2002. The 'Unusual Character' of Holbein's Ambassadors. Artibus et Historiae 23(46): 61-75.

Hansford, D. and D. Collins. 2007. Anamorphic 3D Geometry. Computing 79: 211-203.

Howe, C. and D. Purves. 2005. Perceiving Geometry - Geometrical Illusion Explained by Natural Scene Statistics. New York: Springer.

Hunt, J. L., B.G. Nickel, and C. Gigault. 2000. Anamorphic Images. American Journal of Physics 68(3): 232-237. 
Massey, L. 2007. Picturing Space, Displacing Bodies: Anamorphosis in Early Modern Theories of Perspective. Philadelphia: Penn State Press.

Pérez-Gómez, A. and L. Pelletier. 1977. Architectural Representation and the Perspective Hinge. Cambridge, Massachusetts: The MIT Press.

Pizlo, Z. 2008. 3D Shape: its Unique Place in Visual Perception. Cambridge, Massachusetts: The MIT Press.

Reynolds, M. 2003. Perspectiva Geometrica. Nexus Network Journal 5(1): 137-150.

Schröter, J. 2014. 3D: History, Theory and Aesthetics of the Transplane Image. London: Bloomsbury.

Sharp, J. 1998. Problems with Holbein's Ambassadors and the Anamorphosis of the Skull. In: Bridges Mathematical Connections in Art, Music and Science, 157-165.

Sharp, J. 2010. How to Anamorph. Mathematics Teaching 217: 7-10.

Solina, F. and B. Batagelj. 2007. Dynamic Anamorphosis. In: Proceedings of 4th International Conference on Enactive Interface, 1-4.

Topper, D. 2000. On Anamorphosis: Setting Some Things Straight. Leonardo 33(2): 115-124.

Veltman, K. 1986. Perspective, Anamorphosis and Vision. Marburger Jahrbuch 93-117.

Vesna Stojaković graduated from the program in architecture from Faculty of Technical Sciences, University of Novi Sad. She is Assistant professor at the same Faculty. Her Ph. D. thesis is in the field of image-based modeling applied to cultural heritage. She is doing research and teaching related to geometry, perspective and digital technologies in architecture.

Bojan Tepavčević is an architect. His Ph. D. dissertation about influence of geometric representation of space on contemporary architecture was awarded with International Trimo Research Award in 2011. He is Assistant professor at the University of Novi Sad, where he teaches courses about computational design and architectural representation. His research is published in many research articles and he is the coauthor of the book Architectural Scale Models in the Digital Age: Design, Representation and Manufacturing. 